Fourth International Conference on Sustainable Construction Materials and Technologies http://www.claisse.info/Proceedings.htm

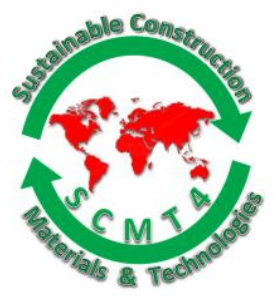

SCMT4

Las Vegas, USA, August 7-11, 2016

\title{
Low-Cost, Robust Microcapsules of Phase Change Materials for Thermal Active Concrete Structures
}

\author{
Jialai Wang*1a, and Fengjuan Liu ${ }^{1 b}$ \\ ${ }^{1}$ Department of Civil, Construction, and Environmental Engineering, The University of Alabama, \\ Tuscaloosa, AL 35406, USA. \\ ${ }^{1 a}$ Email: $<j w a n g @ e n g . u a . e d u>,{ }^{1 b}$ Email: $<\langle f x l i u @ e n g . u a . e d u>$.
}

\begin{abstract}
This study proposes a novel microencapsulation technique for phase change materials (PCMs) so that they can be integrated into concrete to produce thermally active concrete which can be used to reduce energy consumption of buildings. Microencapsulated PCMs (MEPCMs) provide a better utilization of PCMs with building materials. Existing MEPCMs encapsulate PCMs through synthesizing a polymer shell on the surface of PCM droplets. This shell not only is expensive, but also suffers many limitations such as low stiffness/strength, low thermal and chemical stability, high flammability, and low thermal conductivity. To overcome the drawbacks of the existing technologies, this study encapsulates PCMs into hollow fly ash particles, cenospheres. The produced PCM microcapsules are referred to as CenoPCM, which can be directly added into concrete to reduce up to $20 \%$ energy consumption of buildings. A series of experimental studies have been carried out to demonstrate the superior thermal performance of CenoPCM. Prototype thermally active concrete integrated with CenoPCM have been also be developed and characterized to demonstrate the application potential of the CenoPCM in concrete.
\end{abstract}

\section{INTRODUCTION}

The buildings sector of US accounts for approximately $40 \%$ of the United States' primary energy consumption and $39 \%$ of U.S. carbon dioxide emissions. To cope with this challenging situation, efforts are needed to improve energy efficiency of U.S. buildings, which will not only save money for both homeowners and business owners, but also reduce the environmental impacts of energy use. To address this need, this study proposes a novel technique to integrate phase change materials (PCMs) into construction materials to enhance the building energy efficiency through Thermal Energy Storage (TES) and thermal regulation. PCMs change their phase from solid to liquid and vice versa at their phase change temperatures with large amount of energy absorbed or released. Thermal inertia (mass) of the building can be significantly increased by integrating PCMs into construction materials. PCM has been considered as a promising means of TES in terms of narrowing the gap between the peak and off-peak loads of energy/electricity demand, reducing diurnal temperature fluctuations, and utilizing the free cooling at night for day peak cooling load shaving. 
Two methods are commonly used to incorporate PCMs into construction materials: microencapsulation of PCMs and form-stable PCMs composites. In the first method, PCMs are encapsulated within a protective shell with a size of micrometers. The produced microencapsulated PCMs (MEPCMs) can preserve PCMs as long as possible through the heating/cooling cycles. This microencapsulation method increases the heat transfer area, decreases the reactivity of the PCMs, limits the interaction with the construction materials, enhances the low heat conductivity, and facilitates the handling of the PCMs. However, it also suffers a few drawbacks preventing practical applications of the PCMs in construction materials. The protection shell is usually made of polymer, which has very low mechanical stiffness and strength. As a result, the mechanical stiffness and strength of the construction materials can be reduced significantly by adding the PCMs microcapsules. PCMs microcapsules can also been easily broken during the mixing of concrete, leading to leaking of PCMs after melting. The polymeric shell has low chemical and thermal stability. It can be deteriorated by UV light, oxidation, and other aggressive chemicals. It can also lose its stability when temperature exceeds its glass transition temperature. The polymer shells are flammable, and therefore cannot be adopted by US building industry. The thermal conductivity of the polymer shells is very low, making thermal exchange between PCMs inside the shell and outside environment much more difficult.

In the second method, PCMs are first absorbed into porous materials such as light weight aggregates and diatomite particles to form stable composites, which are then added into the construction materials. While in absorbing PCM using porous particles, no protective layers on the surface of the composites are available. As a result, PCMs can still leak from the porous material once the temperature exceeds the phase change materials, leading to reduction or loss of the claimed thermal storage capacity.

To compete with traditional building materials without using PCMs, the above barriers must be removed. To this end, we propose CenoPCM, a PCM microcapsule that uses an industrial waste material, cenospheres, as a protective shell of the PCM core. The expensive process to synthesize the polymer shell used in existing MEPCMs is therefore eliminated. As a result, the cost of this new micro-encapsulation method is only a fraction of the existing MEPCMs. Because cenospheres are used as the shell, CenoPCM also enjoy many other advantages compared with existing MEPCMs:

1) High stiffness/strength: The cenosphere shell of CenoPCM has much higher stiffness/strength than the polymeric shells used in existing MEPCMs. As a result, CenoPCM can endure strong mixing during the manufacturing of the materials, and will not significantly reduce stiffness/strength of the produced materials.

2) High chemical and thermal stability: Since cenospheres are nothing but hollow fly ash particles, they have the same chemical and thermal stability as fly ash. When used in concrete, they can react slowly with the hydration product of Portland cement. This reaction will generate calcium silicate hydrate gel (CSH gel), which can make the CenoPCM shell even stronger.

3) Low flammability: Cenospheres are nonflammable and therefore can reduce the flammability of the organic PCM core, so that CenoPCM can be accepted by US building industry.

4) High thermal conductivity: Since cenospheres are inorganic, their thermal conductivity is much higher than the polymeric shells used in existing MEPCMs, making thermal exchange between PCMs inside the shell and outside environment much easier and faster.

With all these advantages, CenoPCM can eliminate all major barriers preventing application of PCMs in traditional building materials. For example, CenoPCM can be integrated into construction and building materials to improve energy efficiency of U.S. buildings, which account for approximately $40 \%$ of the United States' primary energy consumption and 39\% of U.S. carbon dioxide emissions (EPA 2013). A recent Oak Ridge National Laboratory (ORNL) study indicated that PCM integrated wallboard can result in up to $22 \%$ electricity savings from wall-generated cooling loads (Biswas et 
al. 2014). Another study showed both cooling and heating energy savings are achievable with distributed PCM mixed with cellulose insulation in wall cavities (Biswas and Abhari 2014).

\section{METHODS AND MATERIALS}

In this study, we propose to use cenospheres to encapsulate PCMs so that the technical barriers preventing applications of MEPCMs in building and construction materials can be removed. This new microencapsulation method consists of three steps as shown in figure 1.

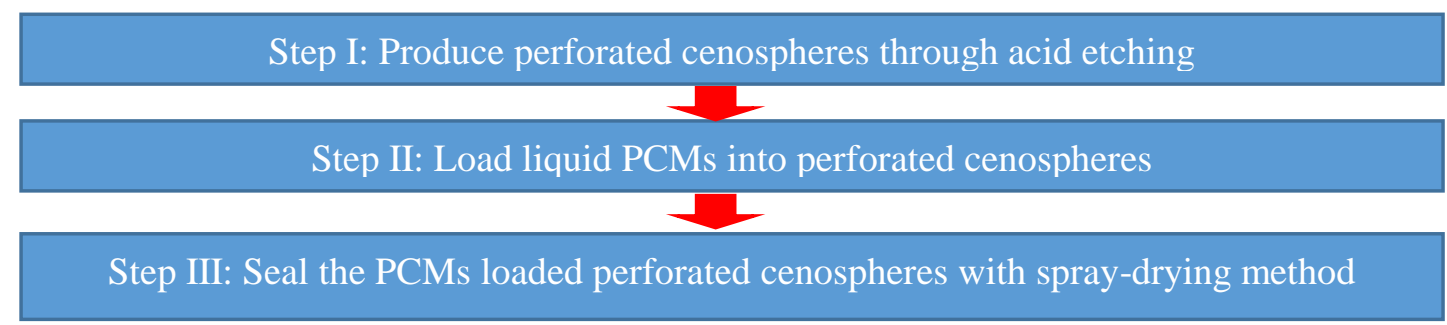

Figure 1. Steps to encapsulate PCMs into cenospheres

In the first step, perforated cenospheres will be produced through acid etching. Cenospheres are hollow fly ash particles generated in coal burning power plants with size ranging from a few micrometers to hundreds of micrometers as shown in figure 2(a). A cenosphere particle has an aluminosilicate shell with high stiffness and strength and a thickness in a few micrometers. The shell has a porous structure formed by gas inclusion and is covered by a glass-crystalline nanosize film, as shown in figure 2(b). Cenospheres have been used in construction materials for decades to produce lightweight materials (Doyoyo and Biju-Duval 2011]. The potential of using the cenospheres as containers for admixtures such as curing water in concrete was noticed by Jensen and Lura (2006) nearly 10 years ago. They stated that "If water-filled, the capsule wall (of cenosphere) could have beneficial pozzolanic properties whereas the water might serve as internal curing water." However, they also pointed out that "no method to induce the water into the particles ... seems to be available." This is because the shell of the cenosphere is covered by a glass-crystalline nanosize film, as shown in figure 2(a), making the inner volume of the cenosphere is inaccessible to admixtures.

The hollow structure of the cenosphere makes it an ideal material to encapsulate PCM. However, to introduce PCM into cenosphere, the glass-crystalline nanosize film on the surface of the cenosphere must be removed. This can be done through acid etching. After removing the nanosize film, perforated cenospheres with very small holes (smaller than $2 \square \mathrm{m}$ ) penetrating through the shell can be produced (figure 2(c)).
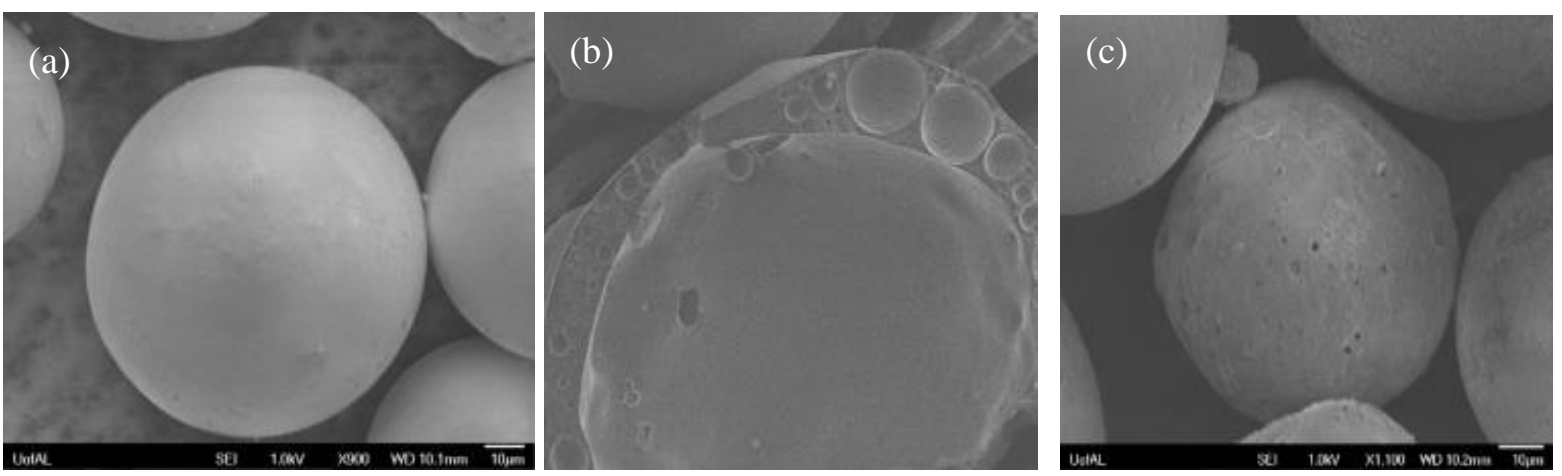

Figure 2 Perforated cenosphere produced by acid etching: (a) impermeable shell before etching; (b) porous shell of the cenosphere; (c) perforated shell after etching with $1.0 \mathrm{M}$ $\mathrm{NH}_{4} \mathrm{~F}-1.2 \mathrm{M} \mathrm{HCl}-\mathrm{H}_{2} \mathrm{O}$ for 2 hours 
In the second step, liquid PCMs are loaded into the perforated cenospheres produced in step I (figure 3 ). In this project, one of the most promising organic PCM, Paraffin waxes ( $n$-alkanes) are loaded. Paraffin waxes have phase-change temperatures of $18-36{ }^{\circ} \mathrm{C}$ and can change phases within this temperature range, making humans feel comfortable. Therefore, they could be used to integrate construction materials to regulate the temperature of the building. Loading Paraffin wax into perforated cenospheres can be simply done by mixing cenospheres in liquid wax to let liquid wax penetrate into the hollow cenospheres. Before loading with liquid wax, cenospheres can float on liquid wax as shown in figure 3(a). After loading, cenospheres settles down at the bottom of the container as shown in figure 3(b). After loading, the extra paraffin wax absorbed on the surface of the cenospheres can be washed by distilled water before it changes its phase to solid, or can be washed using organic solvent such as acetone after it is solidified.

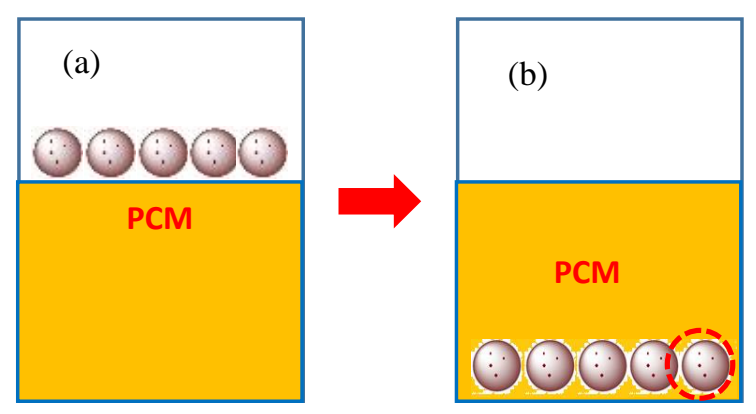

Figure 3. Loading liquid PCM into perforated cenospheres: (a) Before loading, cenospheres float on the liquid PCM; (b) After filled with the PCM, cenospheres settle down at the bottom of the container.

In the third step, a thin layer of silica will be coated on the PCM loaded cenospheres produced in step II to prevent the possible leaking of the liquid PCM, as shown in figure 4 . This coating can be applied by spray-drying method as shown in figure 4. To this end, PCM loaded cenospheres will be first mixed with commercially available silica sol and then sprayed into the drying chamber, in which the silica sol is heated to form a very thin layer of silica. This layer of silica can not only seal the perforated holes on the cenosphere shells, but also strengthen the cenosphere shells.

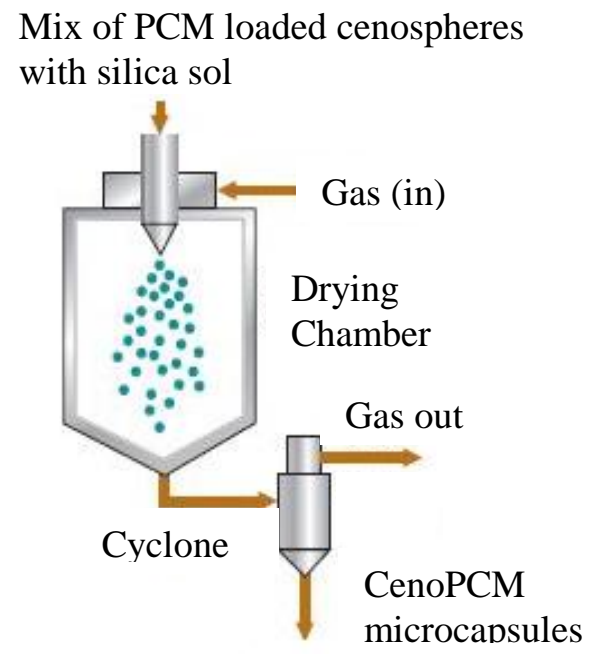

Figure 4: Spray-drying method to a seal the perforated cenospheres with sodium silicate. 


\section{RESULTS AND ANALYSIS}

The produced microcapsules of PCMs are referred to as cenoPCMs since they uses cenospheres as protection shells. Figure 5(c) shows a broken cenoPCM particle. It can be seen clearly that PCM can be successfully loaded into the cenosphere shell. There are a few commercially available MEMCP on the market. BASF and CIBA of UK are two major producers of MEMCP. However, all these available MEMCP use polymeric shells and therefore have the drawbacks aforementioned. Figures 5(a) shows that MEPCM from BASF appear to a large extent deformed and broken, suggesting that a large portion of this MEPCMs cannot survive during the mixing process of concrete (Hunger et al. 2009). Similarly, a large portion of broken MEPCM produced by CIBA can also be identified from figure 5(b) (Dehdezia et al. 2013). Figure 5(d) is the SEM image of the concrete added with 5\% CenoPCM microcapsules. It can be seem that most of these particles are intact, verifying that the proposed CenoPCM has much higher strength than existing polymer based MEPCMs.

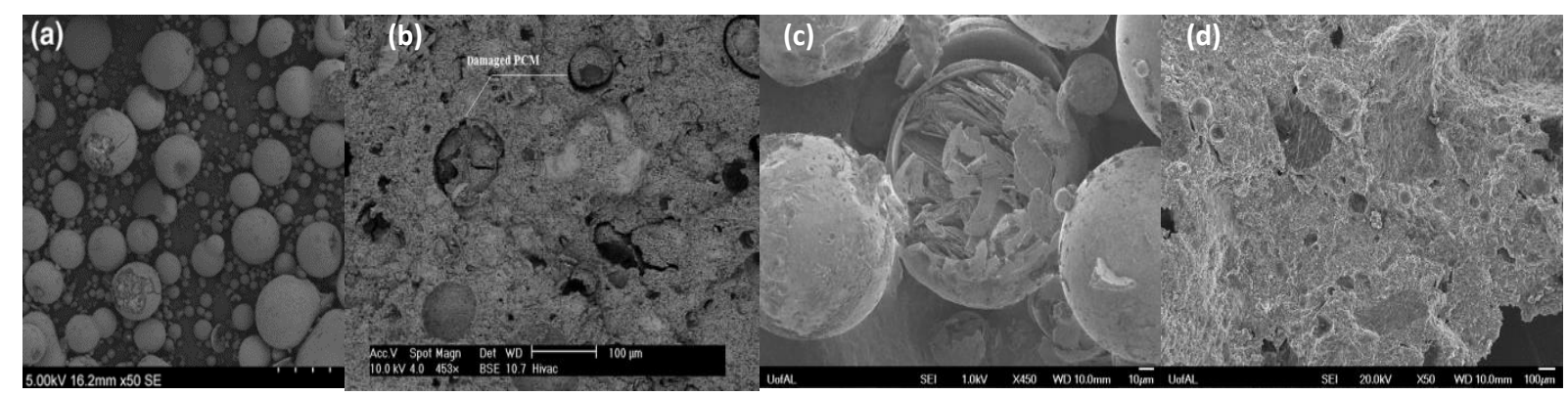

Figure 5 Comparison of cenoPCMs with available commercial products: (a) Micronale, a MEPCM produced by BASF; (b) MEPCM produced by CIBA, UK; (c) cenoPCM proposed in this project; (d) cenoPCM used in concrete

Figure 6(a) shows Differential Scanning Calorimetry (DSC) testing result of the produced CenoPCM. The following procedure was used to perform the DSC measurement: heating/cooling rate $=5^{\circ} \mathrm{C} / \mathrm{min}$., temperature program- scan started at $10^{\circ} \mathrm{C}$ and was complete at $50^{\circ} \mathrm{C}$. A continuous ascending and descending scan was utilized during dynamic DSC testing. Figure 7(a) indicates that the peak melting and freezing temperatures and the latent heats of the CenoPCM are $23.83^{\circ} \mathrm{C}$, $21.57^{\circ} \mathrm{C}, 119.83 \mathrm{~J} / \mathrm{g}, 128.04 \mathrm{~J} / \mathrm{g}$, respectively. Table 1 compares latent heats of the CenoPCM with two commercially available MEPCMs produced by CIBA of UK and BASF of Germany (Zhang and Zhao 2013). Table 1 shows that compare with these commercially available MEPCMs, cenoPCM is superior in latent heat storage capacity.

Figure 6(b) shows thermogravimetry analysis (TGA) result of unsealed CenoPCM. It can be seen that the CenoPCM microparticles starts to lose weight at $150^{\circ} \mathrm{C}$ because PCM decomposes at this temperature. Since the cenospheres were not sealed, decomposed PCM vapor was quickly lost from the perforated cenospheres. This process continued until temperature reached $220^{\circ} \mathrm{C}$. After this temperature, no further weight loss can be observed for the CenoPCM sample, suggesting that all PCM inside the perforated cenospheres had been evaporated. The residual weight of the sample is the weight of the perforated cenosphere shell. From the TGA measurement shown in figure 6(b), we can know that: 1) the PCM accounts for $63.64 \%$ total weight of the produced CenoPCM, suggesting that cenosphere has very high absorption of PCMs; 2) no decomposition occurred in the cenosphere shell within the testing temperature ranges $\left(800^{\circ} \mathrm{C}\right)$, suggesting that cenospheres have much higher thermal stability than polymer shells used in existing MEPCMs. 
CenoPCM removes major barriers preventing wide application of PCMs in building and construction materials because of its low cost, high strength and stiffness, high chemical and thermal stability, low flammability, and high thermal conductivity. Integration of CenoPCM in building materials can save over $20 \%$ energy consumption.
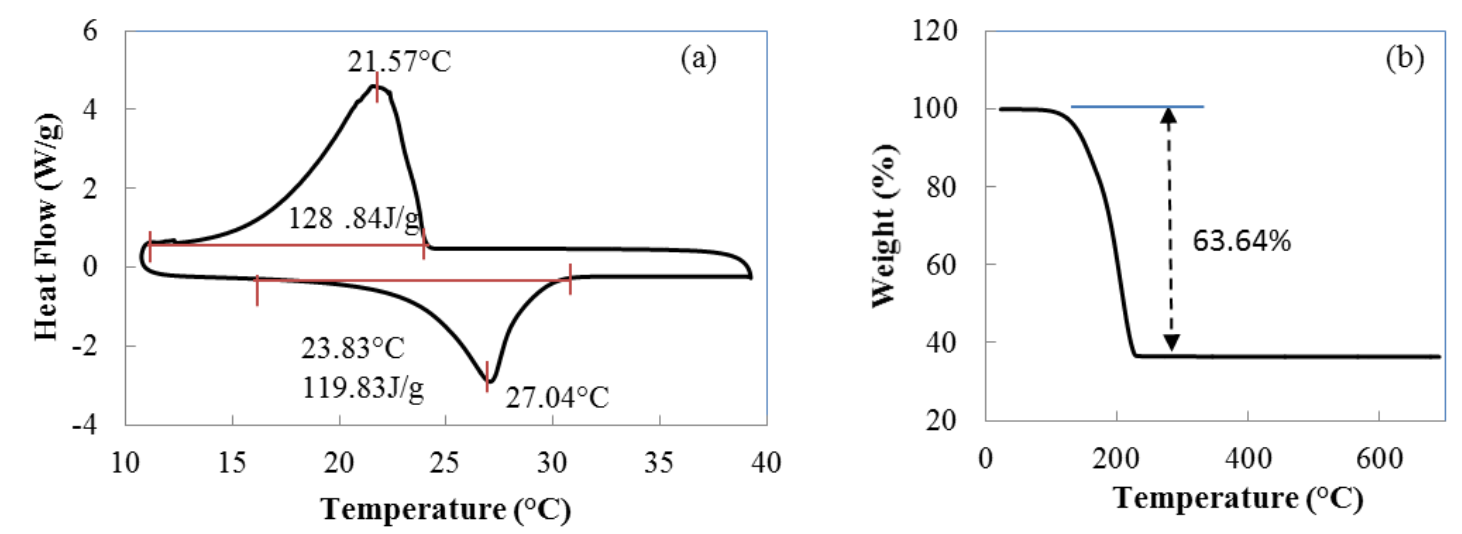

Figure 6. a) DSC measurement of the produced CenoPCM; b) TGA result of unsealed CenoPCM

Table 1. Comparison of latent heat of CenoPCMs with commercially available MEPCMs

\begin{tabular}{|c|c|c|}
\hline Microcapsule name & Melting latent heat $(\mathbf{J} / \mathbf{g})$ & Freezing latent heat $(\mathbf{J} / \mathbf{g})$ \\
\hline CIBA[10] & 102.01 & 106.4 \\
\hline BASF[9] & 96.97 & 101.01 \\
\hline CenoPCM & 119.83 & 128.04 \\
\hline
\end{tabular}

\section{COST ANALYSIS}

The construction industry will see a high switching cost to move from current building materials to PCM-based building materials. However, as with any emerging market, this cost will decrease over time and CenoPCM integrated materials will ultimately scale to current building materials.

Our current technique shows that using one pound of cenospheres can encapsulate $1.5 \mathrm{lb}$ PCM. The cost to encapsulate $1 \mathrm{lb} \mathrm{PCM}=0.67 \mathrm{lb}$ cenospheres at $\$ 0.45 / \mathrm{lb}+1 / 5 \mathrm{lb}$ fluoric acid (used to create perforated cenosphere shells) at $\$ 0.45 / \mathrm{lb}=\$ 0.39 / \mathrm{lb}$. The cost to seal the cenosphere through spray drying $=0.3 \mathrm{lb}$ silica sol at $\$ 0.4 / \mathrm{lb}=\$ 0.12 / \mathrm{lb}$. Therefore, the total manufacturing cost of CenoPCM is $\$ 0.39+\$ 0.12+\$ 0.2$ (labor) $=\$ 0.71 / \mathrm{lb}$. The prices of materials used in the calculation are obtained from http://www.alibaba.com/. The current cost of paraffin wax is $\$ 0.85-\$ 0.91 / \mathrm{lb}$. Accordingly, the cost of one pound PCMs encapsulated with cenosphere is around $\$ 0.71+0.85$ $0.91=\$ 1.56 \sim 1.62 / 1 \mathrm{~b}$, which is much lower than existing MEPCMs (BASF's Micronal price is around $\$ 7 \sim \$ 10 / \mathrm{lb}$ ). According to the calculation of Kosny et al (2012) shown in figure 7, the extra cost of using PCMs in building materials can be paid back through energy saving around 6 years for a single-story ranch house in Bakersfield, CA. With such a short payback period and continuously energy saving up to $20 \%$ every year, it is anticipated the present CenoPCM will find large success on building parts/materials market. 


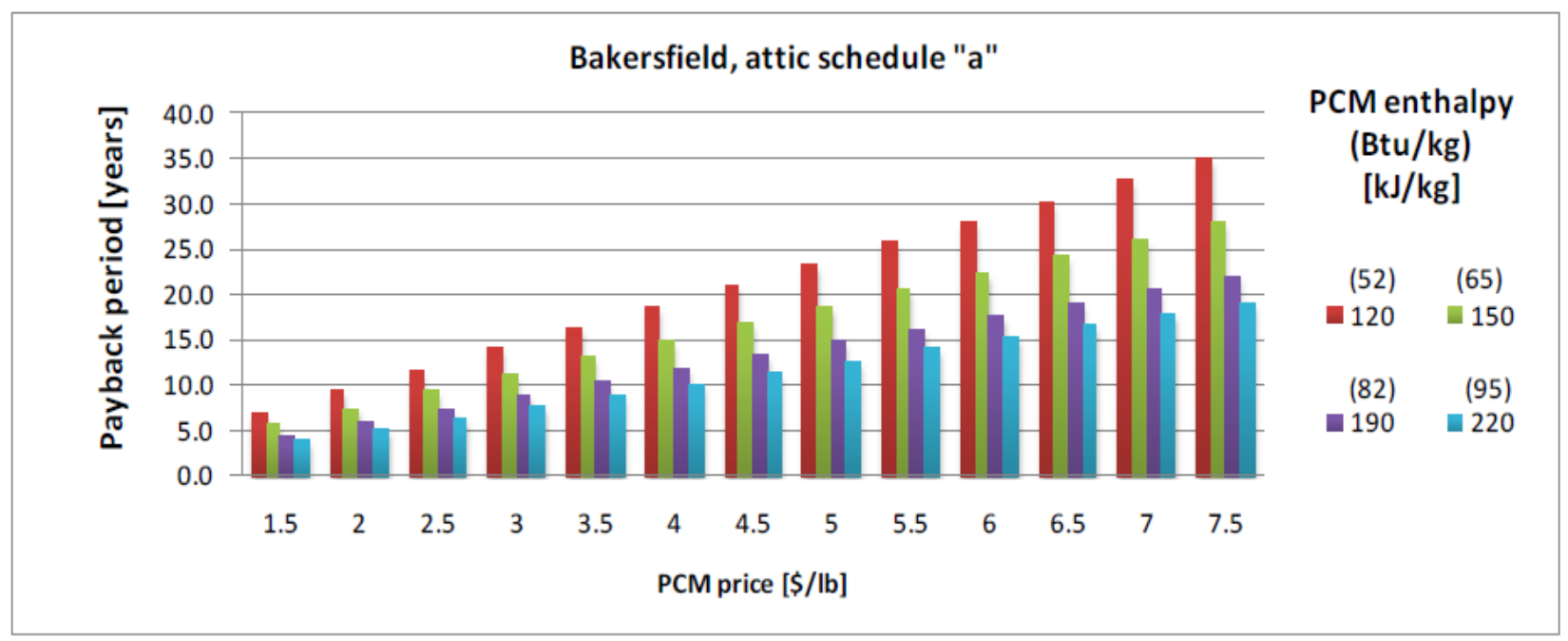

Figure 7. Payback periods for the PCM-enhanced R-30 cellulose insulation configuration installed on the attic floor as a function of the PCM price for a singlestory ranch house in Bakersfield, CA. The external temperature profiles have been defined as "a" $[13]$

\section{CONCLUSION}

A novel technology to encapsulate PCMs into using cenospheres, has been successfully demonstrated in this study. The produced PCM microcapsule, CenoPCM, can be widely adopted in various industrials such as building and construction, energy storage, heating, HVAC, shipping and transportation, etc. for energy saving. CenoPCMs not only overcomes major drawbacks of the currently available polymer-based MEMCPs, but also significantly reduces the cost of the microencapsulation because low-cost industrial waste cenosphere is used.

\section{ACKNOWLEDGEMENTS}

Support for this research project was provided by The University of Alabama and National Science Foundation through CMMI 1000580.

\section{REFERENCES}

Biswas, K., Lu, J., Soroushian, P., and Shrestha, S. (2014). "Combined Experimental and Numerical Evaluation of a Prototype Nano-PCM Enhanced Wallboard.” Applied Energy, 131,517-529.

Biswas K., and Abhari, R. (2014). "Low-Cost Phase Change Material as an Energy Storage Medium in Building Envelopes: Experimental and Numerical Analyses." Energy Conversion and Management, 88, 1020-1031.

Dehdezia, P., Hallb, M., Dawsona, A., and Casey, S. (2013). "Thermal, mechanical and microstructural analysis of concrete containing microencapsulated phase change materials." International Journal of Pavement Engineering, 14, 449-462.

Doyoyo, M., and Biju-Duval,P. (2011). "High Strength Pozzolan Foam Materials and Methods of Making the Same." United States Patent 8,057,594.

EPA. (2013). Monthly Energy Review September 2013, Table 2.1. Washington, DC: Energy Information Agency. 
Hunger. M., Entrop, A., Mandilaras, I., Brouwers, H., and Founti, M. (2009). "The Behavior of SelfCompacting Concrete Containing Micro-Encapsulated Phase Change Materials." Cem Concr Compos 31,731-43.

Jensen, O., and Lura, P. (2006). "Techniques and Materials for Internal Water Curing of Concrete." Materials and Structures, 39, 817-825.

Kosny, J., Shukla, N., and Fallahi, A. (2013). DOE EERE report DE-AC36-08GO28308.

Zhang, G.., and Zhao, Y. (2011). "Thermal and Rheological Properties of Microencapsulated Phase Change Materials." Renewable Energy, 36, 2959-2966. 